\title{
AVES DEL CAMPUS DE LA UNIVERSIDAD NACIONAL AGRARIA LA MOLINA (LIMA-PERÚ) - UNA REVISIÓN DE SU ABUNDANCIA, DISTRIBUCIÓN Y DIVERSIDAD DESDE 1992 AL 2010
}

\section{BIRDS OF THE UNIVERSIDAD NACIONAL AGRARIA LA MOLINA CAMPUS (LIMA-PERU) - A REVISION OF ITS ABUNDANCE, DISTRIBUTION AND DIVERSITY FROM 1992 TO 2010}

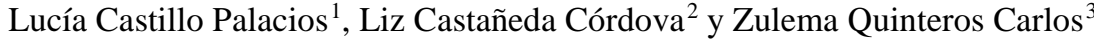

\begin{abstract}
Resumen
El campus de la Universidad Nacional Agraria La Molina (UNALM) Lima - Perú, se presenta como un ecosistema variado y complejo con áreas agrícolas, urbanas y naturales. El presente trabajo es una compilación de tres estudios publicados en los años 1992, 2007 y 2010; y observaciones mensuales realizadas en el año 2010, con el fin de tener un panorama general sobre el cambio de la abundancia, distribución y diversidad de la comunidad de aves presente en la UNALM (1989-2010). Para el año 2010 se obtuvo una lista taxonómica de 48 especies, cuya composición ha variado en comparación con estudios previos, sin embargo, algunas especies de la familia Columbidae y otras como Dives warszewiczi, Pygochelidon cyanoleuca, se mantienen como abundantes desde 1992. La especie con mayor tamaño poblacional y distribución es Zenaida meloda. Por otra parte para el 2010, abril es el mes con mayor abundancia de especies mientras que julio es el menos diverso y abundante, mientras que enero es el mes más diverso posiblemente por la ocurrencia del verano. La UNALM con su variedad de hábitats alberga un mayor número de aves que otras zonas verdes de la ciudad, lo cual la convierte en un espacio importante de diversidad de aves dentro de la ciudad de Lima. Las aves son buenos indicadores de la calidad ambiental y la UNALM es un lugar propicio para el birdwatching. Se recomienda continuar con los monitoreos de la avifauna, así como realizar un análisis detallado sobre la dinámica espacial y temporal.
\end{abstract}

Palabras clave: Avifauna, campus, Lima, UNALM, urbanización, aves urbanas.

\begin{abstract}
The Universidad Nacional Agraria La Molina campus (UNALM) Lima - Peru, presents itself as a varied and complex ecosystem with agricultural, urban and natural zones. This article is a compilation of three past studies published in 1992, 2007 and 2010, and from monthly observations realized in 2010, with the objective of having a general view of the evolution of the bird community in this ecosystem through the years (1989-2011). As for 2010, the taxonomic list includes 48 species. Its composition has changed compared to previous years, however some species from the Columbidae family, and others such as Dives warszewiczi and Pygochelidon cyanoleuca remain as the most abundant since 1989. On the other hand, for 2010, April has the highest abundance and January is the most diverse, possibly due to the occurrence of the summer; July is the least abundant and least diverse as well. UNALM with the variety of its habitats holds a higher number of birds than other green areas of the city; therefore it is an important space for avifauna in the city of Lima. Birds are good indicators of environmental quality and UNALM is a proper space for birdwatching. Deeper analyses on spatio-temporal dynamics and to continue monitoring avifauna are recommended.
\end{abstract}

Key words: Bird community, campus, Lima, UNALM, urbanization, urban birds.

\section{Introducción.}

Actualmente se considera a la Ecología Urbana como la investigación ecológica realizada en ambientes urbanos, con el objetivo de evaluar el planeamiento y manejo de áreas urbanas, con especial interés en las áreas verdes, con el fin para explorar y vislumbrar las diferentes dimensiones de las ciudades que son consideradas ecosistemas (Sukopp, 2002; Endlicher et al., 2007; McDonnell, 2011). En Latinoamérica, el interés por la ecología urbana está creciendo pero aún quedan muchos vacíos por llenar (Ortega-Álvarez \& MacGregor-Fors, 2011; Congreso 
Latinoamericano de Ecología Urbana, 2012), en la actualidad en los campus universitarios de países del neotrópico se tienen listas de especies de aves (Stiles, 1990; Montalvo \& Claver, 1996; Chávez et al., 1999; Maceda \& Kin, 2001; Viñas \& More, 2002; González, 2002; MacGregor-Fors, 2005; Marín, 2005; Muñoz et al., 2007; Ramírez-Albores, 2008; Cardozo et al., 2008; Rodríguez \& Guido, 2008; Chanamé et al., 2010). Por otro lado, es importante reconocer la importancia de estos campus como lugares propicios para el estudio de la presión de la urbanización sobre la avifauna (MacGregor-Fors, 2005). En Perú, específicamente los trabajos en ecología urbana y ornitología urbana son escasos (Takano \& Castro, 2007; Ortega-Álvarez \& MacGregor-Fors, 2011; Castañeda et al., 2013), a pesar que diversos estudios señalan a los ambientes urbanos como propicios para ciertas especies de aves, especialmente aquellas de hábitos generalistas; éstos lugares son idóneos para estudios sobre adaptaciones y nuevos comportamientos (González, 2004b).

Las aves se ven afectadas por diversos factores como la estructura del hábitat, disponibilidad de alimentos, efecto de la estacionalidad, etc. (Isacch \& Martínez, 2001) y cambios en su ambiente, como lo es la urbanización. Es más, éste proceso de urbanización afecta y transforma la estructura física del hábitat; alterando sus procesos ecológicos y evolutivos (Ramírez-Albores, 2008; Juri \& Chani, 2009).

La mayoría de estudios indican que ante el proceso de urbanización se produce una reducción general de la diversidad, abundancia y riqueza de especies de fauna terrestre, en especial las aves, registrando un incremento en la abundancia pero una reducción en su riqueza y diversidad (Blair, 1996; Chace \& Walsh, 2004; Faggi \& Perepelizin, 2006; Almazán-Nuñez \& Hinterholzer-Rodríguez, 2010; Faeth et al., 2011; Nolazco, 2012). Adicionalmente, muchos autores han observado que en las zonas urbanas la riqueza es menor que en las periferias sub urbanas (Faggi \& Perepelizin, 2006). Sin embargo, en algunos casos la riqueza aumenta para zonas con niveles medios de urbanización (Faeth et al., 2011). Por otro lado, unas pocas especies se benefician de esta urbanización y se adaptan a ella, tolerando los cambios que trae consigo, éstas especies suelen ser sedentarias, con una amplia variedad de requerimientos alimenticios (granívoras y omnívoras), altamente sociables y con preferencia de anidamiento en cavidades, que generalmente son estructuras artificiales (Chace \& Walsh, 2004; Faggi \& Perepelizin, 2006; Croci et al., 2008; McGregorFors et al., 2009; Almazán-Nuñez \& HinterholzerRodríguez, 2010).

El distrito de La Molina, al igual que el resto de la ciudad de Lima, presenta una creciente y desordenada urbanización (Ministerio de Vivienda, Construcción y Saneamiento, 2012). Este proceso se ha incrementado notablemente en los últimos años, debido al crecimiento de la población humana, este acelerado crecimiento demográfico reduce los espacios verdes (MacGregor-Fors, 2005; Villacorta et al., 2006; Takano \& Castro, 2007; Nolazco, 2012) y afecta la configuración del hábitat con un consecuente efecto sobre las comunidades biológicas (Marín, 2005; Leveau \& Leveau, 2006; Ortega-Álvarez \& MacGregor-Fors, 2011; González, 2004a; RamírezAlbores, 2008), así como la alteración del paisaje natural. Aquí se ubica el campus de la Universidad Nacional Agraria La Molina - UNALM, es un área distintiva y diferente al resto del distrito donde se localiza, no solo por su extensa área sino también por sus hábitats variados: urbanos, suburbanos agrícolas y ambientes naturalizados, así como granjas, establos y cafetines (Takano \& Castro, 2007; Corrales, 2012), esto lo lleva a albergar una avifauna diversa $\mathrm{y}$ característica.

En 1992, Quinteros inicia el estudio formal de las aves en la UNALM, encontró que la intensidad de uso de los campos de cultivo se incrementa en los últimos estadios del cultivo de maíz, así como que las aves son potenciales plagas a los cultivos de maíz y que la disponibilidad del alimento influencia en el comportamiento de las poblaciones y en la diversidad de la comunidad. En el 2007, Takano \& Castro, realizan un estudio sobre la abundancia y distribución de la avifauna en el campus, así mismo afirman que la UNALM es un lugar apropiado para la observación de aves en la ciudad de Lima. Posteriormente, en el 2010 Takano, identificó aves asociadas a cultivos de maíz y a sus diferentes estadios, encontrando que durante estadios reproductivos se incrementa la diversidad de aves en los campos de cultivo de maíz, así mismo, señala que las aves son responsables de una pérdida considerable de mazorcas de maíz. Finalmente, desde el 2009 el Laboratorio de Ecología de Procesos (LEP) realiza evaluaciones mensuales en el campus de la UNALM, con el fin de registrar la avifauna de la zona; con lo que se intenta dar una descripción general del cambio en abundancia, distribución y diversidad de la comunidad de aves presente en la UNALM basándose tanto en estudios previos y el análisis de datos mensuales del año 2010 obtenidos por el LEP.

\section{Metodología. \\ Área de Estudio.}

El campus de la UNALM (Figura 1) con sus 201 hectáreas se encuentra en el distrito de La Molina, provincia de Lima, departamento de Lima, Perú (12 $05^{\prime}$ S, 7657' O, $251 \mathrm{~m}$ ) (Yoza et al., 2007). Registra una temperatura anual promedio de $20^{\circ} \mathrm{C}$, así como una humedad relativa anual promedio de $81 \%$ y una precipitación total anual de $10 \mathrm{~mm}$ (Observatorio Meteorológico Alexander von Humboldt, 2010). Se encuentra ubicado en la zona de vida Desierto Desecado Subtropical (dd-S) (Holdridge, 1967) y en la ecorregión Desierto del Pacífico (Brack \& Mendiola, 


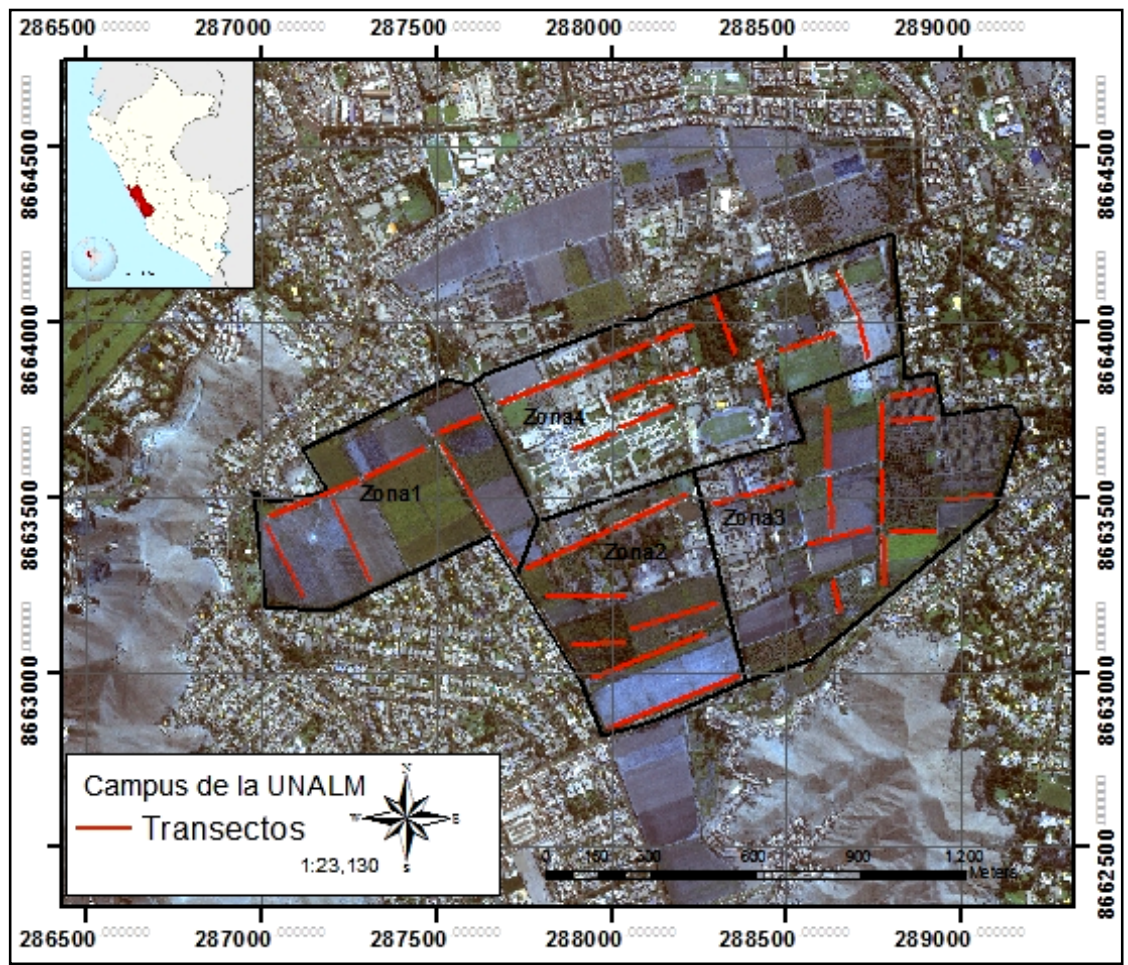

Figura 1. Ubicación de los 37 transectos de conteo de avifauna en el campus de la UNALM Fuente: Laboratorio de Planeamiento Ambiental y Ordenamiento Territorial.

2010). A pequeña escala, observamos un ecosistema complejo y diverso con áreas agrícolas como campos de cultivo de diversas especies (maíz, hortalizas, frutales, etc); zonas naturales con pastos, matorrales y bosques de coníferas; áreas urbanas con construcciones (salones, laboratorios, restaurantes, etc), y jardines, además de pequeños cuerpos de agua y granjas (Takano \& Castro, 2007; Corrales, 2012). Registros fotográficos que datan de 1968 (Municipalidad La Molina, 2012) muestran que para ésta fecha la UNALM presentaba una mayor cantidad de espacios agrícolas que en la actualidad.

Materiales y Métodos.

El presente trabajo es una recopilación de estudios en avifauna publicados previamente en el campus de la UNALM, realizados por Quinteros (1992), Takano \& Castro (2007) y Takano (2010) (Tabla 1); adicionalmente, se utilizaron datos provenientes de los conteos mensuales realizados por el LEP entre enero y diciembre del 2010.

Para el 2010, se realizaron censos mensuales los segundos y/o terceros sábados de cada mes, entre las 7 y 10 am; para lo cual se establecieron 37 transectos de longitudes variadas (100 - 550 metros) distribuidos en toda la UNALM, en base a los transectos propuestos por Quinteros (1992), ubicados en los diversos tipos de hábitat presentes (Figura 1); cada transecto se evaluó en un tiempo promedio de 3 a 5 minutos. Se realizó una división imaginaria del campus en 4 zonas, por lo que cada equipo de observación, conformado por un máximo de tres personas, recorría los diferentes transectos de cada zona, en el mismo orden secuencial en cada

Tabla 1. Resumen sobre estudios previos sobre aves en la UNALM.

\begin{tabular}{|c|c|c|c|}
\hline Estudios & $\begin{array}{ll}\begin{array}{l}\text { Periodo } \\
\text { muestreo }\end{array} & \text { de } \\
\end{array}$ & $\begin{array}{l}\text { Zona } \\
\text { muestreada }\end{array}$ & Tipo de Muestreo \\
\hline $\begin{array}{l}\text { Quinteros } \\
\text { (1992) }\end{array}$ & $\begin{array}{ll}\text { Abril } 1989 & - \\
\text { Abril } 1990 & \end{array}$ & $\begin{array}{l}\text { Campos de } \\
\text { cultivo del } \\
\text { campus }\end{array}$ & Censos por Transectos en Línea \\
\hline $\begin{array}{l}\text { Takano \& } \\
\text { Castro } \\
(2007)\end{array}$ & $\begin{array}{l}\text { Enero } 2001 \text { - } \\
\text { Agosto } 2001 . \\
\text { Setiembre } 2003 \\
\text { - Diciembre } \\
2003\end{array}$ & $\begin{array}{l}\text { Todo el } \\
\text { campus }\end{array}$ & $\begin{array}{l}\text { Enero } 2001 \text { - Agosto 2001: } \\
\text { Método conteo de transecto en } \\
\text { línea (55 transectos). } \\
\text { Setiembre } 2003 \text { - Diciembre } \\
\text { 2003: campus dividido en } 43 \\
\text { zonas, en cada zona se realizaron } \\
\text { avistamientos semanales, se } \\
\text { registró sólo presencia y ausencia } \\
\text { de las especies. }\end{array}$ \\
\hline $\begin{array}{l}\text { Takano } \\
(2010)\end{array}$ & $\begin{array}{l}\text { Agosto } 2006 \text { - } \\
\text { Marzo } 2007\end{array}$ & $\begin{array}{l}\text { Campos de } \\
\text { cultivo de } \\
\text { maíz del } \\
\text { campus }\end{array}$ & $\begin{array}{l}206 \text { conteos: dos personas que se } \\
\text { movían a cada extremo del } \\
\text { campo a velocidad constante, } \\
\text { contando las aves en el medio del } \\
\text { campo. }\end{array}$ \\
\hline
\end{tabular}




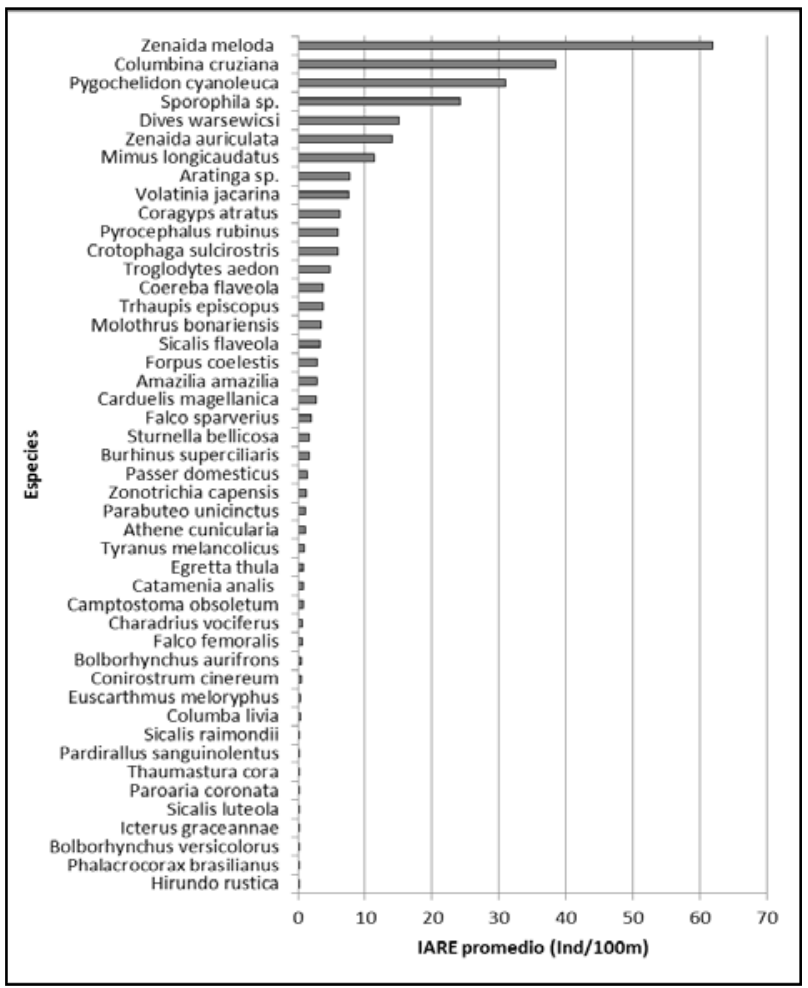

Figura 2. Índices de Abundancias Relativas al Espacio (IARE) Promedio (Ind/100 m) de las especies presentes en el Campus de la UNALM (enero diciembre 2010).

evaluación.

El método empleado fue el Conteo por Transectos en Franja Fija (Ralph et al., 1996) de 30 metros, distancia fijada de acuerdo a los resultados de salidas preliminares. Este método permite registrar un mayor número de individuos y una menor probabilidad de doble conteo en el mismo transecto, para esto también se evitó contar aves que venían volando de la parte posterior del observador (Bibby et al., 1998). Sólo se contabilizaron registros visuales, se descartó trabajar con los auditivos debido a que no se contó con el tiempo suficiente para formar a los censadores.

Análisis de Datos

Se realizó el análisis de los datos colectados desde enero a diciembre del 2010. Se calculó la abundancia relativa, empleando el Índice de Abundancia Relativa al Espacio (IARE), (Quinteros et al., 2002). También se estimó la Diversidad mediante el Índice de Shannon-Wiener (H') (H'=- $\Sigma$ pi $\quad\left(\log _{2} \mathrm{pi}\right)$ ) (Krebs, 1985). Estas medidas han sido utilizadas en muchos estudios en ecología (Margalef, 1991a; Margalef, 1991b; Sánchez, 1992; Véliz, 2002; González, 2004b; Torres et al., 2006; Castañeda et al., 2007; Santana, 2011). También se halló los intervalos de confianza para la abundancia, diversidad y riqueza, que como parte de la estadística inferencial, permitieron observar la variación estacional de dichos parámetros. Adicionalmente para comprobar la distribución de las especies en el campus, según lo observado por Takano \& Castro (2007) con relación a los datos del LEP del 2010 se realizaron mapas de contorno utilizando el programa Surfer 10.

\section{Resultados y discusión.}

Lista Taxonómica de Especies.

En 1992, Quinteros señala 51 especies de aves (24 familias) para la UNALM, este número decrece en el 2007 (Takano \& Castro) donde se describen 46 especies (21 familias). Para el 2010, se tiene una lista de 48 especies (23 familias) (Tabla 2).

Se observa que tanto Takano \& Castro (2007) y los datos del LEP (2010) cubrieron todo el campus y utilizaron métodos similares de censado; sin embargo, Quinteros (1992), aunque presentó la misma metodología que las dos anteriores cubrió solamente campos de cultivo. Finalmente, la lista elaborada por Takano (2010) solo analizó campos de cultivo específicamente de maíz y utilizó una variante del método de censo por transectos.

Para los registros del 2010, 11 especies se encuentran en el Apéndice II de la Categorización de la Convención sobre el Comercio Internacional de Especies Amenazadas de Flora y Fauna Silvestre (CITES). Además, Aratinga erythrogenys se encuentra como Casi Amenazado según el Decreto Supremo $\mathrm{N}^{\circ}$ 004-2014-MINAGRI de especies amenazadas de fauna silvestre del Perú del Ministerio de Agricultura y Riego (MINAGRI, 2014).

Así mismo, 12 especies no se han registrado en el campus en relación a la lista de Quinteros (1992). Para el caso específico de Chordeiles acutipennis se la ha observado ocasionalmente en otras zonas del distrito de La Molina, no en el mismo campus. Es posible explicar la ausencia de Bubulcus ibis por la disminución de campos de cultivos en el campus de la UNALM en comparación con 1992. Por otra parte, a Geranoaetus polyosoma y Circus cinereus se les podía observar en el campus en la década de los 70’s, 80’s y 90’s, sin embargo la reducción de humedales y campos de cultivo- para Circus cinereus, así como el incremento de salones y edificios habrían provocado la disminución de su población, ya que estas especies prefieren hábitats abiertos (Schulenberg et al., 2007). Por otro lado, Parabuteo unicinctus puede haber desplazado a estas rapaces, siendo esta especie dominante en la zona urbana gracias a su introducción desde el norte a finales de los 70's e inicio de la década del 80 (Arnao, L. Comunicación Personal, 6 de febrero, 2014). 

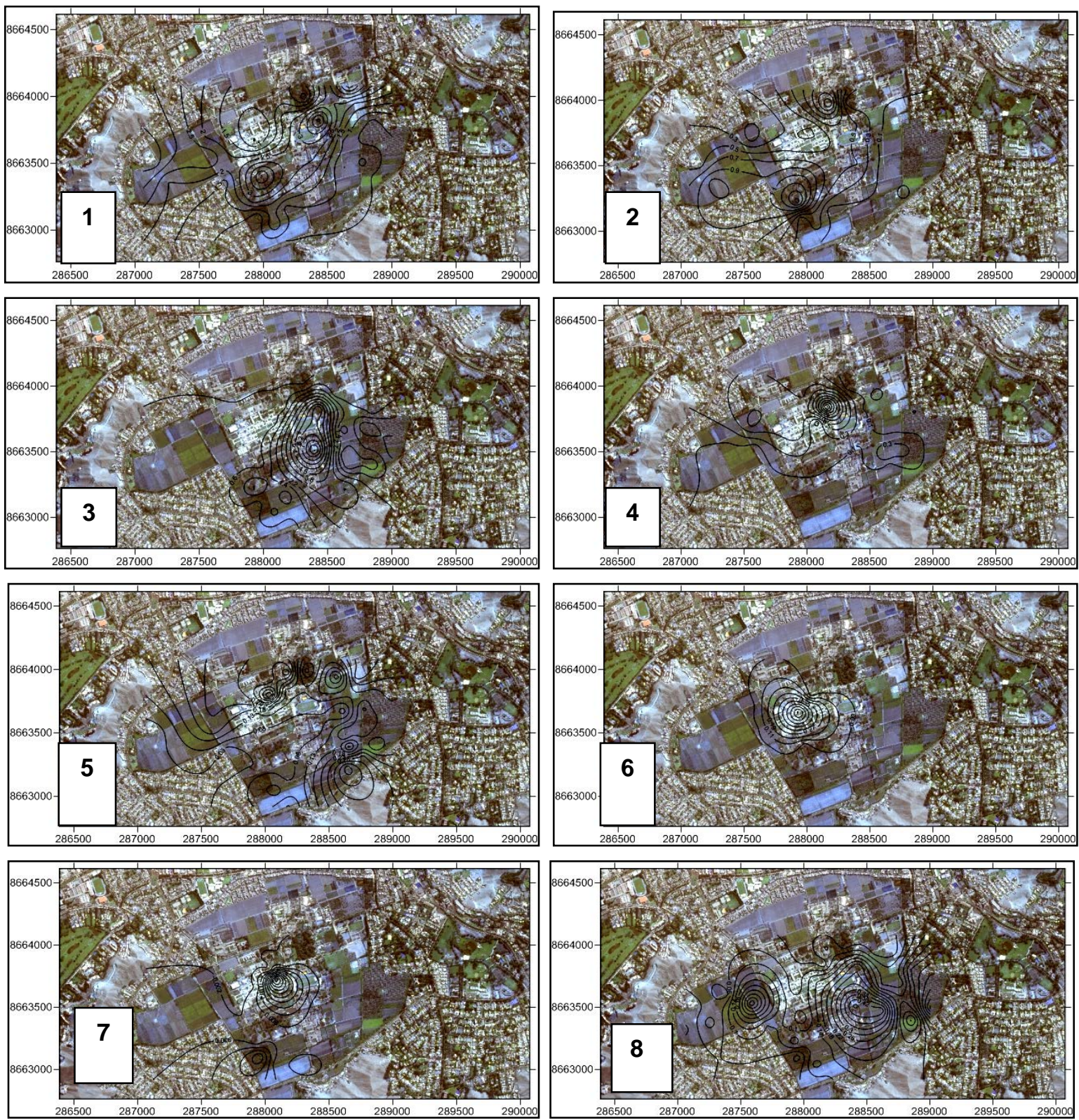

Figura 3. Distribución espacial de especies encontradas en los estudios del 2010 usando isolíneas de abundancia. (1: Zenaida meloda. 2: Dives warszewiczi. 3: Columbina cruziana. 4: Aratinga sp. 5: Pyrocephalus rubinus. 6: Athene cunicularia. 7: Icterus graceannae. 8: Troglodytes aedon).

Abundancia, distribución y diversidad de especies.

En las evaluaciones realizadas entre el 2001 y 2003 (Takano \& Castro, 2007) se halló que las especies más abundantes: Zenaida meloda, Columbina cruziana, Crotophaga sulcirostris y Dives warszewiczi, mientras que las menos abundantes fueron Bulbucus ibis, Hirundo rustica, Myrtis fanny y Parabuteo unicinctus.

Para los conteos realizados en el 2010 por el LEP, el panorama no varía mucho, las colúmbidas siguen siendo las más abundantes: Zenaida meloda (IARE prom: 61.9 Ind/100m) y Columbina cruziana (38.5 Ind/100m) (Figura 4), a ellas se les suma
Pygochelidon cyanoleuca (30.9 Ind/100m), la cual es común en Lima; y Sporophila sp. (24.2 Ind/100m), comunes en áreas de cultivo (Koepcke, 1964; Ortiz, 2012). De las especies con menor abundancia destacan Charadrius vociferus (0.6 Ind/100m), Pardirallus sanguinolentus (0.2 Ind/100m), Icterus graceannae (0.1 Ind/100m) y Phalacrocorax brasilianus $(0.1$ Ind/100m).

La estructura de una comunidad presenta muchas especies con abundancias relativas bajas y pocas especies con abundancias muy altas (Marin, 2005), esto se hace evidente para la comunidad de aves del campus de la UNALM (Figura 2). 


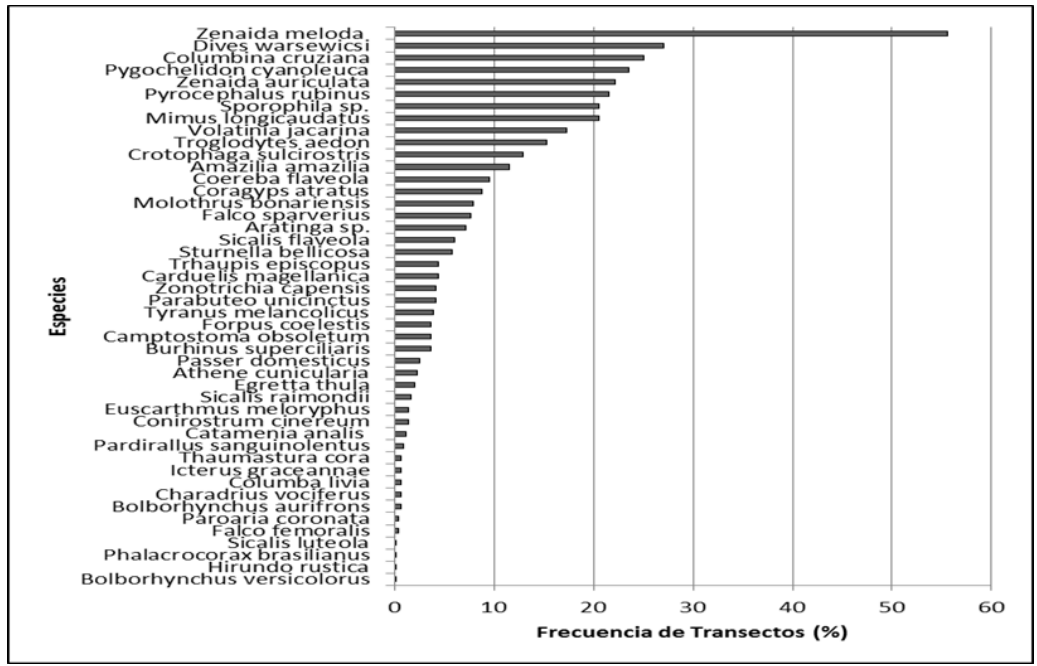

Figura 4. Porcentaje de transectos promedio utilizados por las especies presentes en la UNALM.

En cuanto a la distribución de especies dentro del campus, según Takano \& Castro (2007), las especies con un mayor rango de distribución son: Zenaida meloda, Columbina cruziana, Dives warszewiczi, Pyrocephalus rubinus y Troglodytes aedon, mientras que las especies con menor rango de distribución son: Bubulcus ibis, Icterus graceannae, Aratinga sp., Athene cunicularia y Glacidium brasilianum. Para el año 2010, este panorama tampoco no varía mucho. La especie que ocupa el $55.6 \%$ del total de los transectos es la Zenaida meloda (Figura 4), además la Figura 3.1 muestra las isolíneas de abundancia se distribuyen ampliamente en todo el campus, tanto en zonas agrícolas como urbanas, posiblemente por ser generalista y mejor adaptada a la urbanización (Marín, 2005; Castro \& Takano, 2007); por otro lado Columbina cruziana se concentra más en zonas agrícolas y granjas para el 2010 (núcleos en dichas zonas), con un $25 \%$ de utilización de transectos del total. Dives warszewiczi, también tiene un rango de distribución considerable pero con una aparente preferencia por campos de cultivo como lo muestran la isolíneas de la Figura 3.2 y con un $27.1 \%$ de utilización de transectos (Figura 4). Así mismo, con una distribución amplia está Pyrocephalus rubinus, común en zonas con terrenos arbustivos (Koepcke, 1964; Schulenberg et al., 2007), se observan isolíneas de abundancia en la zona urbana del campus con jardines y arbustos (Figura 3.5) y con menor intensidad en zonas de cultivos de frutales (mandarinas y lúcumas), además de tener un $21.4 \%$ de utilización de transectos. Finalmente, Troglodytes aedon, tiene una distribución moderadamente amplia en el campus (Figura 3.8; Figura 4), se puede observar mayor concentración en zonas con campos de cultivo y zonas arbustivas, siendo este último su hábitat preferido (Schulenberg et al., 2007).
De las especies con rango de distribución más agregado, para el 2010 tenemos que Athene cunicularia presenta una distribución agregada en la zona urbana entre salones y jardines, donde se han observado nidos, se sabe que esta especie anida en huecos que escarba en el suelo (Koepcke, 1964). Para el caso de Icterus graceannae con una distribución y abundancia restringida se le puede observar en la zona urbana y algunas áreas rurales (avistamientos ocasionales). Por otro lado, a pesar que Aratinga sp. según Takano \& Castro (2007) presenta una distribución más agregada podemos observar que para el 2010 presenta una distribución más amplia en zonas urbanas y parte de campos de cultivo (Figura 3), es posible que Aratinga sp. aproveche una mayor cantidad de espacio que en años anteriores, debido a los recursos que puede encontrar en el campus (alimento, lugares de reposo y nidificación).Todas estas especies también presentan pocos transectos utilizados con respecto al total (Figura 4).

En la Figura 6, se observa tres espacios diferentes en la UNALM, Campos de Cultivo (Zona 1) presenta con zonas cultivadas con predominancia de maíz, yuca, y cultivos diversos, así como malezas; Campos de Frutales, conformados por arbustos de mandarina y lúcuma. Finalmente, la Zona Urbana, área compuesta por edificios y jardines. Las especies predominantes en cada uno de los espacios, refuerza lo mencionado anteriormente, se puede observar por ejemplo que Zenaida meloda es predominante en los tres tipos de formaciones vegetales, así como que Mimus longicaudatus abunda más en la zona urbana.

Para el año 2010, el Îndice de Abundancia Relativa al Espacio, presenta mayores valores para el mes de abril y segundos picos en junio, con una gran variabilidad durante estos meses en los 37 transectos; los valores mínimos se dan en julio y octubre (Figura 5A). Los picos altos se deben a la alta abundancia de Zenaida meloda durante estos meses, la cual es la especie más abundante para el periodo evaluado; mientras que los meses de baja abundancia se explican por la baja abundancia de especies dominantes como Zenaida meloda, Columbina cruziana y Pygochelidon суаnoleuca. Se notan diferencias entre algunos meses, especialmente Octubre, el cual de acuerdo a sus intervalos de confianza es diferente a los meses de marzo y abril, es necesario continuar las evaluaciones, para diferenciar patrones por un efecto estacional o por un evento puntual para ese año. 


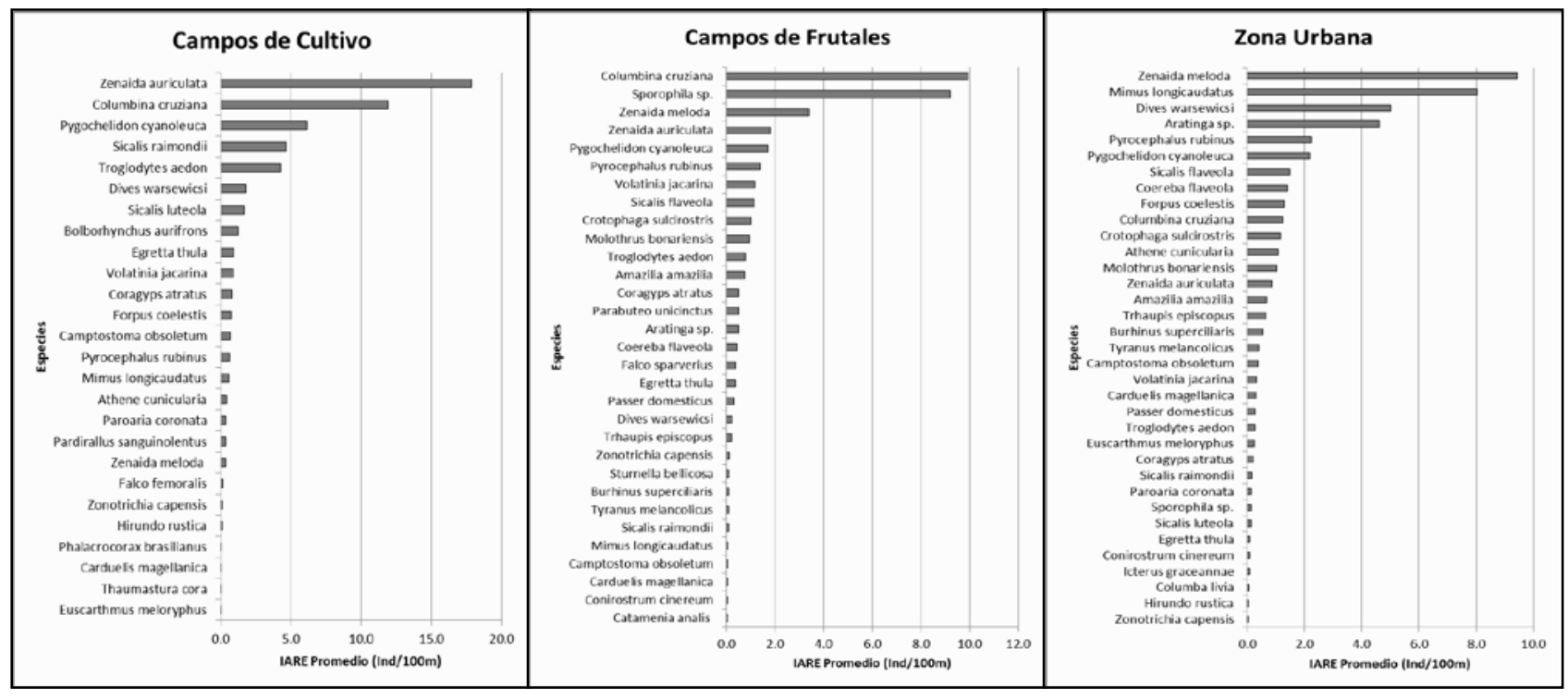

Figura 5. Caracterización de avifauna en diferentes espacios en términos de uso y estructura vegetal.

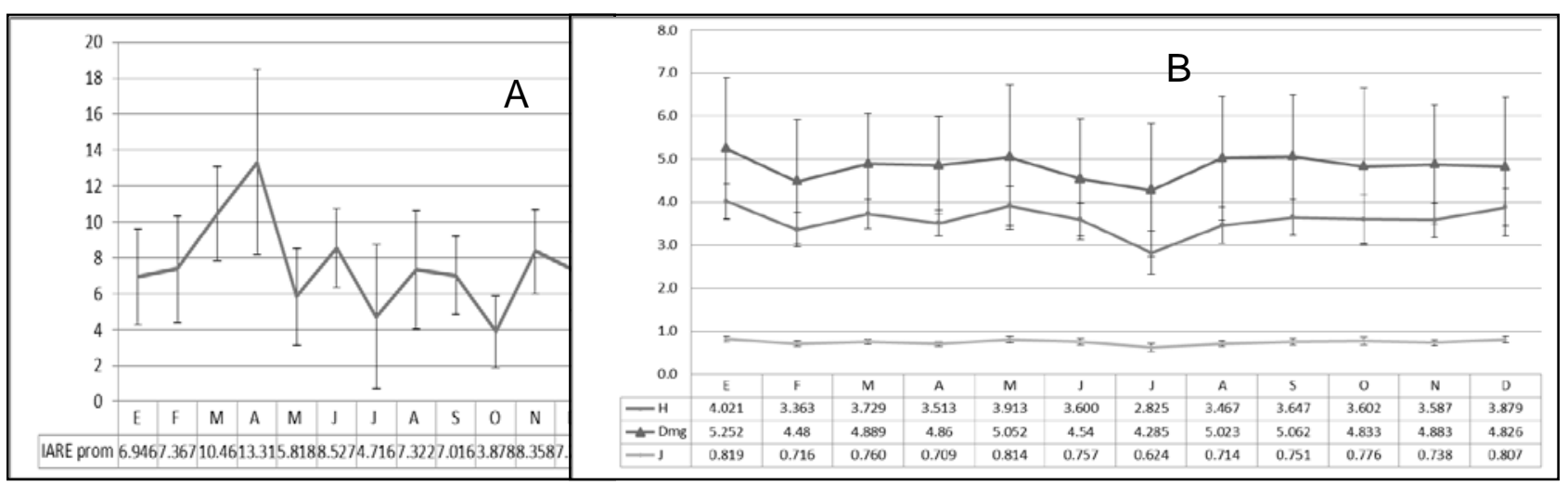

Figura 5A. Variación temporal del Índice de Abundancia Relativa al Espacio (IARE: Ind/100m). Figura 5.B: Índice de Shannon-Wiener (H’, bits), Riqueza de Margalef (Dmg) y Equitativilidad (e) durante el periodo enero diciembre 2010.

Para la diversidad, el Índice de Shannon- Wiener se mantiene entre los 2.825 bits como valor mínimo para julio y 4.021 bits como valor máximo para enero, a pesar de no existir una estacionalidad marcada para esta zona, es posible que exista una mayor diversidad de aves durante los meses de verano y primavera septiembre a febrero (González, 2004b) (Figura 5.B). El Índice de diversidad de Margalef, presenta un comportamiento similar al Índice de Shannon Wiener, con su punto más bajo en julio y el más alto en enero. Finalmente, la Equidad no varía mucho durante los meses analizados.

5.3. Especies asociadas a campos de cultivo

Quinteros (1992), encontró 22 especies asociadas a campos de cultivo, de las cuales 16 estaban directamente relacionadas con el cultivo de maíz. La especies más abundantes fueron Zenaida meloda, Columbina cruziana, Aratinga sp., S. bellicosa, Psilopsiagon aurifrons, Carduelis magellanica,
Passer domesticus Sporophila sp., Molothrus bonariensis y Dives warszewiczi (Santana, 2011).

Takano en el 2010 incrementa la lista a 38 especies asociadas a los campos de cultivo de maíz (Tabla 2). Solo se comparan ambos estudios porque fueron realizados en campos de cultivos de maíz. Para el 2010 (LEP) se observa un número esperado de 36 especies en los campos de maíz; ambos estudios presentan resultados similares posiblemente por ser los más recientes. En 1992, Quinteros concluye también que el alimento es un factor limitante y por lo tanto determina los patrones de distribución de las poblaciones; el cultivo de maíz se presenta como un modelador de la distribución de las aves, especialmente en sus últimos estadios, donde la actividad de las aves se intensifica.

Especies raras, invasoras y plagas.

En el campus de la UNALM se han registrado entre los años 1989 y 2011, mediante avistamientos ocasionales y censos, especies raras o poco comunes 
para la zona, ya sea por visitas ocasionales atraídas por algún hábitat específico o, por ser especies victimas de tráfico ilegal para ser usadas como mascotas (Koepcke, 1964; Quinteros 1992; Schulenberg et al., 2007; Takano \& Castro, 2007; Takano 2010) (Tabla 3).

Tabla 3. Especies poco comunes registradas en la UNALM

\begin{tabular}{ll}
\hline Nombre Científico & Nombre Común \\
\hline Anairetes reguloides & Torito Garganta Negra \\
Circus cinereus & Gavilán de Campo \\
Dolichonyx oryzivorus & Chambergo \\
Gallinula chloropus & Polla de Agua \\
Molothrus oryzivorus & Tordo Gigante \\
Pardirallus sanguinolentus & Gallineta común \\
Phalacrocorax brasilianus & Cushuri \\
Phalcoboenus megalopterus & Chinalinda \\
Saltator striatipectus & Saltator Rayado
\end{tabular}

Del mismo modo también existen varias especies consideradas exóticas que se han adaptado (Tabla 4) y establecido no sólo en el campus de la UNALM, sino también en varias zonas de la ciudad de Lima (González, 2004a), gracias a los recursos que esta le ofrece.

Tabla 4. Especies exóticas registradas en la UNALM

\begin{tabular}{ll}
\hline Especie & Zona de origen \\
\hline Aratinga erythrogenys & Costa norte \\
Brotogeris versicolorus & Amazonia central \\
Burhinus superciliaris & Costa norte \\
Forpus coelestis & Costa norte \\
Icterus graceannae & Costa norte Paraguay, \\
& Bolivia, Brata \\
Paroaria coronata & Uruguay, Brasil y \\
& Argentina \\
Thraupis episcopus quaesita & Noroeste \\
\hline
\end{tabular}

\section{Conclusiones.}

El campus de la UNALM, presenta una comunidad de aves conformada por 48 especies; dicha variedad y abundancia existe gracias a que la UNALM es un espacio altamente heterogéneo en términos de hábitat, presentado espacios con campos de cultivo de diversas especies (maíz, frutales, hortalizas, etc.) así como zonas con construcciones rodeadas de jardines. Por otro lado, también existen granjas y establos, cuerpos de agua, etc. Esta riqueza de hábitats, especialmente las áreas cultivadas y zonas naturalizadas, contribuyen a que la avifauna se diversifique en comparación al resto del distrito (prioritariamente urbano), así como con otras áreas verdes de tamaño comparable. Además, en la UNALM éstas especies encuentran fuentes variadas de alimento, zonas de reposo y anidamiento, etc.

La riqueza de especies en la UNALM es mayor que las registradas en otras zonas y parques de considerable tamaño debido tal vez a su variedad de hábitats; como "Lima Golf Club" (48 ha) con 34 especies (Ramírez \& González, 2001) y el Parque "El Olivar” (23 ha) con 29 especies (Takano \& Castro, 2007); siendo así la UNALM un lugar con una alta riqueza de aves e ideal para la práctica de observación de aves en la ciudad (birdwacthing).

Esto hace importante el mantenimiento de lugares como el campus de la UNALM, dentro de ciudades en constante crecimiento, donde el campo y las áreas verdes están siendo reemplazadas por concreto; así mismo la heterogeneidad de hábitats de la UNALM, puede funcionar como amortiguador para contrarrestar el efecto de la masificación urbana.

Por último, se recomienda continuar con los monitoreos de la avifauna en el campus de la UNALM, así como realizar un análisis más detallado sobre la dinámica de la avifauna, incluyendo un análisis espacial y temporal de dicho ecosistema. Así mismo, continuar con estudios sobre los hábitos alimenticios de las aves, en vista a que en los últimos años, se ha observado que el comportamiento alimenticio de las aves difiere del esperado según la bibliografía. Observaciones ocasionales, en espacios urbanos del campus como cafetines, restaurantes y kioskos, han registrado especies como Zenaida meloda, D. warszewiczi, Mimus longicaudatus, Sicalis flaveola, Crotophaga sulcirostris, entre otras, consumiendo papas fritas, salchichas, trozos de pollo cocido y golosinas; incluso se han reportado individuos de la especie Dives warszewiczi ingiriendo vísceras de pollo crudas.

\section{Agradecimientos.}

Al Laboratorio de Ecología de Procesos por el apoyo brindado y facilidades para el análisis de los datos, así como a todos los asistentes, estudiantes y egresados que apoyaron durante el periodo de investigación. Al Laboratorio de Planeamiento Ambiental y Ordenamiento Territorial por brindarnos la imagen satelital y al Observatorio Meteorológico Alexander von Humboldt por dar acceso a los datos de temperatura ambiental del campus para el año 2010. Un agradecimiento especial a los revisores anónimos por sus valiosas sugerencias y recomendaciones que permitieron mejorar el presente artículo.

\section{Literatura citada.}

Almazán-Núñez R.C. \& Hinterholzer-Rodríguez A. 2010. Dinámica Temporal de la Avifauna en un parque urbano de la ciudad de Puebla, México. Huitzil Revista Ornitológica Mexicana. 11(1): 26-34.

Arnao L. 2014. Rapaces en la UNALM (Correspondencia personal). Perú.

Bibby C., Jones M. \& Marsden S. 1998. Expedition field Techniques. Bird surveys. London: Expedition Advisory Centre.

BirdLife International. 2013. BirdLife Taxonomic Checklist Version 6. Consultado 25 de Febrero de 2014. 
Disponible

http://www.birdlife.org/datazone/info/taxonomy

Blair R.B. 1996. Land use and avian species diversity along an urban gradient. Ecological Aplications. 6 (2): 506519.

Brack A. \& Mendiola C. 2010. Ecología del Perú. Editorial Bruño. 496 p.

Cardozo G., Beltzer A. \& Collins P. 2008. Variación primavero-estival de la diversidad y abundancia de la comunidad de aves en la Reserva Ecológica de la Ciudad Universitaria U.N.L. “El Pozo”. Miscelánea. 17 (2): 367-386.

Castañeda L., Arellano G. \& Sánchez E. 2007. Efecto de una quema controlada en los artrópodos epígeos de pasturas en la SAIS Túpac Amaru, Junín - Perú. Ecología Aplicada. 6 (1,2): 47-58.

Castañeda L.Z., Arnao L.M., Castillo L.N., Álvarez S.C., Quinteros D.Z. \& Caro C.C. 2013. PERU 100-110 p. en Ecología Urbana: Experiencias en Latinoamérica. Eds. MacGregor-Fors \& Ortega-Álvarez. es

Chanamé J., Angulo E., Carmona M. \& Puse E. 2010. Avifauna del Campus de la Universidad Nacional Pedro Ruiz Gallo. Lambayeque. Octubre 2007-Abril 2008. Ciencia, Tecnología y Humanidad. 1 (1): 47-58

Chace J.F. \& Walsh J.J. 2004. Urban effects on native avifauna: a review. Landscape and Urban Planning. Disponible en: www.elsevier.com/locate/landurbaplan

Chávez C., Balmaceda J., Viñas P. \& Otero D. 1999. Importancia del campus de la Universidad Nacional de Piura en la conservación de aves silvestres. Res. III Jornada Nacional de Ornitología. Calca, Cuzco, Perú.

Comercio Internacional de Especies Amenazadas de Flora y Fauna Silvestre.2014. Base de datos de especies de la CITES (en línea). Consultado 10 may. 2014. Disponible en http://www.cites.org/esp/disc/species.php

Congreso Latinoamericano de Ecología Urbana: Desafíos y escenarios de desarrollo para las ciudades latinoamericanas. (1, 2012, Buenos Aires, Argentina). 2012. (Libro de Resúmenes). Eds. A C Herrero. Buenos Aires, Argentina. $1^{\text {a }}$ ed. UNGS. 287 p.

Corrales M.S. 2012. Percepción de la Comunidad Universitaria sobre los Vertebrados Urbanos en la Universidad Nacional Agraria La Molina. Tesis Título de Biólogo. Lima. Perú. Universidad Nacional Agraria La Molina. 105 p.

Croci S., Butet A. \& Clergeau P. 2008. Does urbanization filter birds on the basics of their biological traits? The Condor 110 (2): 223-240.

Endlicher W., Langner M., Hesse M., Mieg H.A., Kowarik I., Hostert P., Kulke E., Nützmann G., Schulz M., Van der Meer E., Wessolek E \& Wiegand C. 2007. Urban Ecology- definitions and concepts en Shrinking Cities: Effects on Urban Ecology and challenges for urban development. Langner M., Endlicher W. (eds). Peter Lang.

Faeth SH., Bang C. \& Saari S. 2011. Urban biodiversity: patters and mechanisms. Annals of the New York Academy of Sciences. 1223: 69-81.

Faggi A. \& Perepelizin P.V. 2006. Riqueza de aves a lo largo de un gradiente de urbanización en la ciudad de Buenos Aires. Revista del Museo Argentino de Ciencias Naturales. 8(2): 289-297.
González O. 2002. Distribución y dispersión del mielero (Coereba flaveola, Aves: Coerebidae) en la ciudad de Lima, Perú. Ecología Aplicada. 1(1): 115-116.

González O. 2004a. Ecología de aves urbanas en un parque de la Ciudad de Lima. Tesis de Maestría. Universidad Nacional Mayor de San Marcos. Lima, Perú.

González O. 2004b. Variación Espacio-Temporal de la diversidad de Aves Urbanas en un Área verde de la ciudad de Lima. Revista Científica Dilloniana 4 (1): 116-117.

Holdridge LR. 1967. Life Zone Ecology. Tropical Science Center. San José, Costa Rica. 149 pp.

Isacch J.P. \& Martínez M.M. 2001. Estacionalidad y relaciones con la estructura del hábitat de la comunidad de aves de pastizales de Paja Colorada (Paspalum uadrifarium) manejados con fuego en la provincia de Buenos Aires, Argentina. Ornitología Neotropical. 12: 345-354.

Juri M.D. \& Chani J.M. 2009. Variación estacional en la composición de las comunidades de aves en un gradiente urbano. Ecología Austral. 19 (3): 175-184. Disponible en http://www.scielo.org.ar/scielo.php?script=sci_arttext\&p id=S1667-782X2009000300001

Koepcke M. 1964. Las Aves del Departamento de Lima. Talleres Gráfica Morson S.A. Lima.

Krebs C.J. 1985. Ecology: The Experimental Analysis of Distribution and Abundance. Third Edition. Harper and Row. New York. 800p.

Leveau C. \& Leveau L. 2006. Ensambles de aves en calles arboladas de tres ciudades costeras del sudeste de la Provincia de Buenos Aires, Argentina. Hornero. 21 (1): 25-30.

Maceda J. \& Kin M.S. 2001. Lista de Aves de la Facultad de Agronomía de Santa Rosa (La Pampa) y sus alrededores. Rev. Fac. Agron. UNLPam. 12 (1): 21-30.

Magurran A. 1989. Diversidad Ecológica y su Medición. Editorial Vedra Barcelona.

Margalef R. 1991a. Ecología. Séptima Edición. Ediciones Omega S.A. Barcelona.

Margalef R. 1991b. Teoría de los Sistemas Ecológicos. Primera Edición. Publicaciones de la Universitat de Barcelona.

Marín O. 2005. Avifauna del Campus de la universidad del Quindío. Boletín Sao. 15(2): 42-59.

MacGregor-Fors I. 2005. Listado Ornitológico del Centro Universitario de ciencias Biológicas y Agropecuarias, Universidad de Guadalajara, Jalisco, México: un espacio suburbano. Huitzil Revista de Ornitología Mexicana. 6: 1-6.

MacGregor-Fors I., Ortega-Álvarez R. \& Schondube J. 2009. On the Ecologica Quality of Urban System : An Ornithological Perspective. En: Graber D. and Birmingham K. (Eds.) Urban Planning in the 21st Century, Nova Science Publishers, New York.

McDonnell M. J. 2011. The history of urban ecology: An ecologist's perspective. Páginas 5-13. En: Urban Ecology: Patterns, Processes and Applications. Niemelä J., Breuste J., Elmqvist T., Guntenspergen G., James P., McIntyre N.E. (eds). Oxford University Press, Oxford.

MINAGRI. 2014. Decreto Supremo N N 004-2014MINAGRI. Consultado 20 de Julio de 2014. Disponible en

http://siar.regionloreto.gob.pe/index.php?accion=verEle 
mento\&idElementoInformacion=570\&idTipoElemento= 3

Ministerio de Vivienda, Construcción y Saneamiento. 2012. Compendio Estadístico. Situación Habitacional. Lima, Perú. Consultado 19 de Agosto de 2013. Disponible en http://fenix.vivienda.gob.pe/Compendio2011/

Moreno C. 2001. Métodos para medir la biodiversidad .Volumen I. GORFI S.A. Zaragoza.

Montalvo N. \& Claver J. 1996. Guía de aves silvestres de las Facultades de Agronomía y de Ciencias Veterinarias de la Universidad de Buenos Aires. Rev. Fac. Agron. UBA. 7: 191-216.

Municipalidad La Molina. 2012. 50 años. La Molina, disfrutando mi hogar. 1962-2012. Aza Graphic Peru S.A.C, Lima.

Muñoz M., Fierro-Calderón K. \& Rivera-Gutiérrez H. 2007. Las aves del campus de la Universidad del Valle, una isla verde urbana en Cali, Colombia. Ornitología Colombiana. 5: 5-20.

Nolazco S. 2012. Diversidad de aves silvestres y correlaciones con la cobertura vegetal en parques y jardines de la ciudad de Lima. Boletín Informativo UNOP. 7 (1): 4-16.

Observatorio Meteorológico Alexander Von Humboldt. 2010. Datos de Temperaturas Promedio - 2010 Universidad Nacional Agraria La Molina.

Ortega-Álvarez R. \& MacGregor-Fors I. 2011. Dusting-off the file: A review of knowledge on urban ornithology in Latin America. Landscape and Urban Planning. 101: 110.

Ortiz C. 2012. Alimentación Nocturna de la Golondrina azul y blanca Pygochelidon cyanoleuca (Hirundinidae) en el Perú. The Biologist (Lima) 10 (1): 74-75.

Plengue M. 2012. List of the Birds of Perú. Boletín UNOP.

Quinteros Z. 1992. Determinación de los Patrones de uso Temporal y Espacial de los Cultivos de Maíz por las Aves Granívoras de la UNALM. Tesis para optar al Título de Bióloga. Universidad Nacional Agraria La Molina. Lima, Perú.

Quinteros Z., Sánchez E. \& Tovar H. 2002. Relación entre la abundancia relativa y densidad real entre 3 poblaciones de aves. Ecología Aplicada. 1(1): 95-104.

Ralph C.J., Geupel G.R., Pyle P., Martin T.E., De Sante D.F. \& Milá B. 1996. Manual de Métodos de Campo para el Monitoreo de aves terrestres. Gen. Tech. Rep. PSW-GTR-159. Albany,CA: Pacific Southwest Research Station, Forest Service, U.S. Department of Agriculture.

Ramírez-Albores J. 2008. Comunidad de aves de la Facultad de Estudios Superiores Zaragoza Campus II, UNAM, Ciudad de Mexico. Huitzil Revista de Ornitología Mexicana. 9 (2). 12-19.

Ramírez D. \& González O. 2001. Análisis de dos Métodos de Muestreo para el Cálculo de la Diversidad de Especies de Aves Terrestres en la Ciudad de Lima, Perú. Biota. 100: 114-123.

Rodríguez C. \& Guido I. 2008. Lista preliminar de la Avifauna de la Ciudad Universitaria Carlos Monge
Alfaro de la Universidad de Costa Rica, San Ramón 2006-2008. Inter Sedes. 9 (16): 11-22.

Sánchez E.H. 1992. Diversidad y Estabilidad de Comunidades de Aves en la Reserva Nacional de Lachay. Tesis para optar el Grado Magister Scientiae. Lima. Perú. Universidad Nacional Agraria La Molina. $111 \mathrm{p}$.

Sánchez E., Quinteros Z. \& Falero M. 1998. Avances en la evaluación y manejo poblacional de Psitácidos (loros y pericos) en la Región Grau. 381 - 395pp. En: Bosques Secos y Desertificación. Memorias del Seminario Internacional. Proyecto Algarrobo INRENA. Lambayeque.

Santana C.A. 2011. Patrones de Variación Espacio Temporal Diurno de las Aves en el Aeropuerto Internacional Jorge Chávez durante los años 2009-2010 y descripción de su relación con las operaciones de vuelos de aeronaves. Tesis para optar el título de Biólogo. Lima. Perú. Universidad Nacional Agraria La Molina. $132 \mathrm{p}$.

Schulenberg T., Stotz D., Lane D., O’Neill J. \& Parker T. 2007. Birds of Peru. Princenton University Press. New Jersey - USA.

Stiles F. 1990. La Avifauna de la Universidad de Costa Rica y sus alrededores a través de veinte años (1968.1989). Revista Biología Tropical. 38 (2b): 361-368.

Sukopp H. 2002. On the early history of urban ecology in Europe. Preslia, Praha. 74: 373-393.

Takano F. 2010. Diversidad de aves y estimación del daño que causan sobre la producción de maíz en el campus de la UNALM. Tesis para optar al título de Biólogo. Universidad Nacional Agraria La Molina. Lima, Perú.

Takano F. \& Castro N. 2007. Avifauna en el Campus de la Universidad Nacional Agraria La Molina (UNALM), Lima-Perú. Revista de Ecología Aplicada. 4 (1,2): 149154.

Torres M., Quinteros Z. y Takano F. 2006. Variación Temporal de la Abundancia y diversidad de aves Limícolas en el Refugio de Vida Silvestre Pantanos de Villa. Lima, Perú. Ecología Aplicada 5 (1,2): 119-125.

Véliz C. 2002. Resiliencia en Comunidades de aves en la Reserva Nacional de Lachay luego del evento EL Niño (1997-98)”. Tesis para optar el título de Biólogo. Lima. Perú. Universidad Nacional Agraria La Molina. 128 p.

Villacorta S., Chamba G., Carlotto V. \& Fídel L. 2006. Atlas ambiental de Lima Metropolitana: Mapas de susceptibilidad en el ordenamiento territorial. XIII Congreso Peruano de Geología. Resúmenes Extendidos Sociedad Geológica del Perú. 171-174 p.

Viñas P. \& More A. 2002. Avifauna del Campus de la Universidad de Piura : Del Desierto al Bosque. Boletín de Lima. 127: 77-85.

Yoza L., Chávez O., David S. \& Lezcano L. 2007. Ruido Ambiental en el campus de la Universidad Nacional Agraria La Molina. Anales Científicos (UNALM). 68 (2): 18-26. 
Tabla 2. Lista taxonómica comparativa de especies presentes de aves del campus de la UNALM en Quinteros (1992), Takano \& Castro (2007), Takano (2010) y evaluaciones del LEP (2010)- Orden taxonómico según Plengue (2012).

\begin{tabular}{|c|c|c|c|c|c|c|}
\hline Nombre Científico & Nombre Común* & Familia & $\begin{array}{l}\text { Quinteros } \\
\text { (1992) }\end{array}$ & Takano \& Castro (2007) & Takano (2010) & LEP -2010 \\
\hline Phalacrocorax brasilianus & Cushuri & Phalacrocoracidae & & & & $\mathrm{X}$ \\
\hline Bubulcus ibis & Garza Bueyera & & $\mathrm{X}$ & $\mathrm{X}$ & & \\
\hline Egretta thula & $\begin{array}{l}\text { Garza } \\
\text { Pequeña, }\end{array}$ & Ardeidae & $\mathrm{X}$ & & & $\mathrm{X}$ \\
\hline Coragyps atratus & $\begin{array}{ll}\text { Gallinazo } & \text { de } \\
\text { Cabeza Negra } & \end{array}$ & Cathartidae & $\mathrm{X}$ & $\mathrm{X}$ & & $\mathrm{X}$ \\
\hline Buteo platypterus & $\begin{array}{l}\text { Aguilucho de Ala } \\
\text { Ancha }\end{array}$ & & $\mathrm{X}$ & & & \\
\hline Geranoaetus polyosoma & Aguilucho Común & & $\mathrm{X}$ & & & \\
\hline Parabuteo unicinctus ${ }^{c}$ & $\begin{array}{l}\text { Gavilán Oscuro } \\
\text { Acanelado }\end{array}$ & Accipitriaae & $\mathrm{X}$ & $X$ & & $\mathrm{X}$ \\
\hline Circus cinereus & Gavilán de Campo & & & $\mathrm{X}$ & & \\
\hline Falco femoralis $^{c}$ & Halcón Perdiguero & & $\mathrm{X}$ & & & $\mathrm{X}$ \\
\hline Falco sparverius $^{c}$ & $\begin{array}{l}\text { Cernícalo } \\
\text { Americano }\end{array}$ & Falconidae & $X$ & $\mathrm{X}$ & $\mathrm{X}$ & $\mathrm{X}$ \\
\hline Pardirallus sanguinolentus & Gallineta común & Rallidae & $\mathrm{X}$ & & $\mathrm{X}$ & $\mathrm{X}$ \\
\hline Burhinus superciliaris & Huerequeque & Burhinidae & $\mathrm{X}$ & $\mathrm{X}$ & $\mathrm{X}$ & $\mathrm{X}$ \\
\hline Charadrius vociferus & $\begin{array}{l}\text { Chorlo Doble } \\
\text { Collar }\end{array}$ & Charadriidae & & $\mathrm{X}$ & $\mathrm{X}$ & $\mathrm{X}$ \\
\hline Metriopelia ceciliae & Cascabelita & & $\mathrm{X}$ & & & \\
\hline Zenaida auriculata & Rabiblanca & & $\mathrm{X}$ & $\mathrm{X}$ & $\mathrm{X}$ & $\mathrm{X}$ \\
\hline Zenaida meloda & Cuculí & & $\mathrm{X}$ & $\mathrm{X}$ & $\mathrm{X}$ & $\mathrm{X}$ \\
\hline Columbia livia & Paloma Doméstica & columbidae & & $\mathrm{X}$ & & $\mathrm{X}$ \\
\hline Columbina minuta & Alfarerita & & $\mathrm{X}$ & $\mathrm{X}$ & & \\
\hline Columbina cruziana & Tortolita Peruana & & $\mathrm{X}$ & $\mathrm{X}$ & $\mathrm{X}$ & $\mathrm{X}$ \\
\hline Ara severus & $\begin{array}{l}\text { Guacamaya de } \\
\text { Frente Castaña** }\end{array}$ & & & & $\mathrm{X}$ & \\
\hline Aratinga erythrogenys ${ }^{C N T}$ & $\begin{array}{l}\text { Cotorra Cabeza } \\
\text { Roja }\end{array}$ & & $\mathrm{X}$ & $\mathrm{X}$ & $\mathrm{X}$ & $\mathrm{X}$ \\
\hline Aratinga wagleri & Cotorra de Wagler & & $\mathrm{X}$ & $\mathrm{X}$ & $\mathrm{X}$ & \\
\hline Aratinga mitrata & Cotorra Mitrada** & Psittacidae & & & $\mathrm{X}$ & \\
\hline Forpus coelestis $^{C}$ & $\begin{array}{l}\text { Periquito } \\
\text { Esmeralda** }\end{array}$ & & $\mathrm{X}$ & $\mathrm{X}$ & $\mathrm{X}$ & $\mathrm{X}$ \\
\hline Psilopsiagon aurifrons ${ }^{C}$ & $\begin{array}{l}\text { Perico } \\
\text { Cordillerano }\end{array}$ & & $\mathrm{X}$ & $\mathrm{X}$ & $\mathrm{X}$ & $\mathrm{X}$ \\
\hline Brotogeris versicolurus ${ }^{C}$ & Perico Andino & & $\mathrm{X}$ & $\mathrm{X}$ & & $\mathrm{X}$ \\
\hline Crotophaga sulcirostris & Guardacaballo & Cuculidae & $\mathrm{X}$ & $\mathrm{X}$ & $\mathrm{X}$ & $\mathrm{X}$ \\
\hline Athene cunicularia ${ }^{C}$ & $\begin{array}{l}\text { Lechuza de los } \\
\text { Arenales }\end{array}$ & Strigidae & $\mathrm{X}$ & $\mathrm{X}$ & & $\mathrm{X}$ \\
\hline Glaucidium brasilianum & Paca Paca & & $\mathrm{X}$ & $\mathrm{X}$ & & $\mathrm{X}$ \\
\hline Chordeiles acutipennis & $\begin{array}{l}\text { Chotacabras } \\
\text { Trinador }\end{array}$ & Caprimulgidae & $\mathrm{X}$ & & & \\
\hline Streptoprocne zonaris & $\begin{array}{l}\text { Vencejo de Collar } \\
\text { Blanco }\end{array}$ & Apodidae & $\mathrm{X}$ & & & \\
\hline Aeronautes andecolus & Vencejo Andino & & $\mathrm{X}$ & & & \\
\hline Rhodopis vesper & $\begin{array}{l}\text { Picaflor Cola } \\
\text { Ahorquillada }\end{array}$ & & & $\mathrm{X}$ & & $\mathrm{X}$ \\
\hline 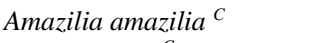 & Amazilia Costera & Trochilidae & $\mathrm{X}$ & $\mathrm{X}$ & $\mathrm{X}$ & $\mathrm{X}$ \\
\hline Myrtis Fanny ${ }^{C}$ & Picaflor de Fanny & & $\mathrm{X}$ & $\mathrm{X}$ & & $\mathrm{X}$ \\
\hline Thaumastura cora ${ }^{C}$ & Picaflor de Cora & & $\mathrm{X}$ & $\mathrm{X}$ & & $\mathrm{X}$ \\
\hline Camptostoma obsoletum & $\begin{array}{l}\text { Mosqueta } \\
\text { silbadora }\end{array}$ & & $\mathrm{X}$ & $\mathrm{X}$ & $\mathrm{X}$ & $\mathrm{X}$ \\
\hline Anairetes reguloides & $\begin{array}{l}\text { Torito Garganta } \\
\text { Negra }\end{array}$ & & & & & $\mathrm{X}$ \\
\hline Euscarthmus meloryphus & $\begin{array}{l}\text { Mosqueta } \\
\text { Copetona }\end{array}$ & Tyrannidae & $\mathrm{X}$ & & & \\
\hline Myiophobus fasciatus & $\begin{array}{l}\text { Mosqueta } \\
\text { Modesta }\end{array}$ & & $\mathrm{X}$ & & & \\
\hline Pyrocephalus rubinus & Turtupilín & & $\mathrm{X}$ & $\mathrm{X}$ & $\mathrm{X}$ & $\mathrm{X}$ \\
\hline Tyrannus melancholicus & $\begin{array}{l}\text { Pepite } \\
\text { Golondrina Azul y }\end{array}$ & & $\mathrm{X}$ & $\mathrm{X}$ & & $\mathrm{X}$ \\
\hline Pygochelidon cyanoleuca & $\begin{array}{l}\text { Blanca o Santa } \\
\text { Rosita } \\
\text { Golondrina }\end{array}$ & Hirundinidae & $\mathrm{X}$ & $\mathrm{X}$ & $\mathrm{X}$ & $\mathrm{X}$ \\
\hline Hirundo rustica & $\begin{array}{l}\text { Tijerita } \\
\text { Migratoria }\end{array}$ & & & $\mathrm{X}$ & & $\mathrm{X}$ \\
\hline Petrochelidon rufocollaris & Golondrina & & $\mathrm{X}$ & & & \\
\hline
\end{tabular}


Tabla 2. Lista taxonómica comparativa de especies presentes de aves del campus de la UNALM en Quinteros (1992), Takano \& Castro (2007), Takano (2010) y evaluaciones del LEP (2010)- Orden taxonómico según Plengue (2012).

\begin{tabular}{|c|c|c|c|c|c|c|}
\hline Nombre Científico & Nombre Común* & Familia & $\begin{array}{l}\text { Quinteros } \\
\text { (1992) }\end{array}$ & Takano \& Castro (2007) & Takano (2010) & LEP -2010 \\
\hline & Cuevas & & & & & \\
\hline Troglodytes aedon & Cucarachero & Troglodytidae & $\mathrm{X}$ & $\mathrm{X}$ & $\mathrm{X}$ & $\mathrm{X}$ \\
\hline Mimus longicaudatus & Chisco & Mimidae & $\mathrm{X}$ & $\mathrm{X}$ & $\mathrm{X}$ & $\mathrm{X}$ \\
\hline Thraupis episcopus & Violinista & & $\mathrm{X}$ & $\mathrm{X}$ & & $\mathrm{X}$ \\
\hline Conirostrum cinereum & Mielero Gris & Thraupidae & $\mathrm{X}$ & $\mathrm{X}$ & $\mathrm{X}$ & \\
\hline Paroaria coronata & $\begin{array}{l}\text { Cardenal de Cresta } \\
\text { Roja*** }\end{array}$ & IIIIdupiude & & & $\mathrm{X}$ & \\
\hline Catamenia analis & $\begin{array}{l}\text { Corbatita Pico de } \\
\text { Oro }\end{array}$ & & & $\mathrm{X}$ & $\mathrm{X}$ & $\mathrm{x}$ \\
\hline Sicalis flaveola & $\begin{array}{l}\text { Chiringue } \\
\text { Azafranado** }\end{array}$ & & $\mathrm{X}$ & $\mathrm{X}$ & $\mathrm{X}$ & $\mathrm{X}$ \\
\hline Sicalis raimondii & $\begin{array}{l}\text { Chiringue de } \\
\text { Raymondi }\end{array}$ & & & & & $\mathrm{X}$ \\
\hline Sicalis luteola & Chiringue Común & & & & & $\mathrm{x}$ \\
\hline Volatinia jacarina & Saltapalito & & $\mathrm{X}$ & $\mathrm{X}$ & $\mathrm{X}$ & $\mathrm{X}$ \\
\hline Sporophila simplex & Espiguero Simple & Emberizidae & $\mathrm{X}$ & $\mathrm{X}$ & $\mathrm{X}$ & $\mathrm{X}$ \\
\hline Sporophila telasco & $\begin{array}{l}\text { Espiguero } \\
\text { Corbatón }\end{array}$ & 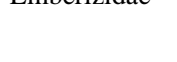 & $\mathrm{X}$ & $\mathrm{X}$ & $\mathrm{X}$ & $\mathrm{X}$ \\
\hline Sporophila luctuosa & $\begin{array}{l}\text { Espiguero Negro y } \\
\text { Blanco** }\end{array}$ & & & & $\mathrm{X}$ & \\
\hline Tiaris obscurus & Espiguero Pardo & & $\mathrm{X}$ & & & \\
\hline Sporophila peruviana & $\begin{array}{l}\text { Pico Grueso } \\
\text { Gorrión }\end{array}$ & & $\mathrm{X}$ & & & \\
\hline Zonotrichia capensis & $\begin{array}{l}\text { Americano } \\
\text { Pichisanka }\end{array}$ & & & $\mathrm{X}$ & $\mathrm{X}$ & $\mathrm{X}$ \\
\hline Coereba flaveola & $\begin{array}{l}\text { Mielerito } \\
\text { Común** }\end{array}$ & Coerebidae & & $\mathrm{X}$ & $\mathrm{X}$ & $\mathrm{X}$ \\
\hline Molothrus bonariensis & Tordo Parásito & & $\mathrm{X}$ & $\mathrm{X}$ & $\mathrm{X}$ & $\mathrm{X}$ \\
\hline Molothrus orzyvorus & Tordo Gigante** & & & & $\mathrm{X}$ & \\
\hline Dives warszewiczi & Tordo Grande & & $\mathrm{X}$ & $\mathrm{X}$ & $\mathrm{X}$ & $\mathrm{X}$ \\
\hline Icterus graceannae & $\begin{array}{l}\text { Bolsero de Filos } \\
\text { Blancos** }\end{array}$ & Icteridae & & $\mathrm{X}$ & & $\mathrm{X}$ \\
\hline Sturnella bellicosa & Pecho Colorado & & $\mathrm{X}$ & $\mathrm{X}$ & $\mathrm{X}$ & $\mathrm{X}$ \\
\hline Dolichonyx orzyvorus & Chambergo & & & & $\mathrm{X}$ & \\
\hline Sporagra magellanica & $\begin{array}{l}\text { Jilguero de Cabeza } \\
\text { Negra }\end{array}$ & Fringillidae & $\mathrm{X}$ & $\mathrm{X}$ & $\mathrm{X}$ & $\mathrm{X}$ \\
\hline Passer domesticus & Gorrión Europeo & Passeridae & $\mathrm{X}$ & $\mathrm{X}$ & & $\mathrm{x}$ \\
\hline TOTAL DE ESPECIES & & & 51 & 46 & 38 & 48 \\
\hline
\end{tabular}

*Nombre común según Koepcke, 1964. **Nombre común según Plengue, 2012. ***Nombre común según BirdLife International, 2013.

C Apéndice II de la Categorización CITES

${ }^{\mathrm{NT}}$ Casi Amenazado (especies amenazadas de fauna silvestre del Perú del MINAGRI, 2014).

${ }^{1}$ Laboratorio de Ecología de Procesos. Universidad Nacional Agraria La Molina. Av. La Molina s/n Apartado 12-056 Lima12 Perú. Correo electrónico: 1_castillo_p@hotmail.com

${ }^{2}$ Laboratorio de Ecología de Procesos. Universidad Nacional Agraria La Molina. Av. La Molina s/n Apartado 12-056 Lima12 Perú. Correo electrónico: lizcastaneda@lamolina.edu.pe

${ }^{3}$ Laboratorio de Ecología de Procesos. Universidad Nacional Agraria La Molina. Av. La Molina s/n Apartado 12-056 Lima12 Perú. Correo electrónico: zquinteros@lamolina.edu.pe 